\title{
A CAD Paradigm to Produce Blow Moulds for Decorated PET Bottles
}

\author{
V. Gulati \\ Department of Mechanical \\ Engineering, GJ University of Science \\ and Technology \\ Hisar, India
}

\author{
P. Tandon \\ Department of Mechanical \\ Engineering, PDPM-Indian Institute of \\ IT, Design \& Manufacturing Jabalpur, \\ India
}

\begin{abstract}
A CAD paradigm is presented to produce blow moulds for Poly Ethylene Terephthalate (PET) bottles decorated with ornamental patterns. This work integrates design and manufacturing of decorated blow moulds which, in turn, are utilized to blow PET bottles. The parametric geometric modeling techniques are employed to customize a CAD system, tailoring to suit the requirements of the bottle making industry with in integrated environment.
\end{abstract}

\section{Keywords}

Blow moulds, PET bottles, CAD, patterns.

\section{INTRODUCTION}

PET bottles used in the packaging of drinks, food stuffs and personal care products are manufactured by inflating a hot and hollow thermoplastic preform inside a closed blow mould made in two halves. Each mould half consists three sections (top, middle and bottom) having cavities corresponding to the three portions of the bottle. The shape of the PET bottle is completely dictated by the shape of the mould cavity, as depicted in Figure 1. This work is emphasized on decoration of the middle section of mould with patterns in order to provide a level of appeal to customers towards the product filled in the bottle and demand for novelty in PET bottles.

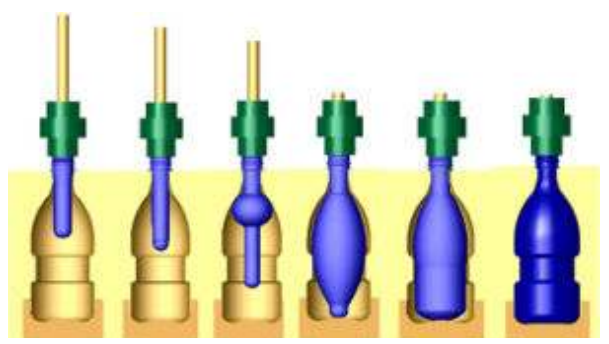

Figure 1. Inflation in blow forming process

Most of the PET bottles consists a very common design of circumferential ribs. Such types of bottle designs give almost similar appearances, thus decreases aesthetic and commercial value of the product filled in the bottle. It is desirable to adorn the beverage bottles in order to provide a level of artistic appearance which is one of the requirements of the bottle making industry.
This work proposes a modeling approach to generate decorated blow moulds for PET bottles within an integrated environment. The integration of parametric geometric modeling techniques and basic modules of proprietary $\mathrm{CAD} / \mathrm{CAM}$ software is created to produce bottle moulds from initial design with minimal modeling time.

\section{REVIEW OF LITERATURE}

The various methods of parametric modeling techniques have been employed in the integrated design and manufacture of blow moulds for decorated bottles. A customized application programme has been developed to create a library of "bottledesign" features and to integrate design and manufacture of a blow mould of bottle within proprietary CAD/CAM software named Duct5 [1]. A blow-moulding prototype system was presented wherein a geometric model of hollow plastic container of desired contour is first designed and then used to generate a geometric model of the corresponding mould cavity using CAD software [2]. An invention has been created to develop a blow moulded transparent containers provided with a decoration comprising label or sleeve [3]. A method of designing and manufacturing a blow moulded container having highly artistic sculptural relief has been presented which graphically designs a container skin shape, creates 2-D artwork designs, adds 3-D relief to the artwork designs, and applies the artwork to the container skin to develop a desired container for further making of blow moulds which, in turn, are utilized to blow containers [4]. A work has been also presented which designs and develops of a surface scanning system to capture surface data in a point-cloud data file, which can be post processed by a CAD system to construct a surface model of the scanned object. The created CAD model can be used to facilitate the design and making of thermoforming moulds for forming plastic sheets for packaging consumer products [5].

\section{ORNAMENTAL PATTERNS}

A parametric voxel based approach is presented for designing ornamental patterns. CAD is used as a tool to generate a set of voxel elements as building blocks and to arrange the selected ones into some definite order to shape an ornamental pattern. The various shapes of voxels are defined by parameterized geometry and attributes. The rules on application to the voxel elements lead to the generation of a range of patterns that can be replicated on bottle moulds [6]. 


\subsection{Modeling of Voxel Elements}

Voxel elements are created using the translational sweep which uses 2D entities to a sketch cross section. Some of the voxel elements are shown in Figure 2.

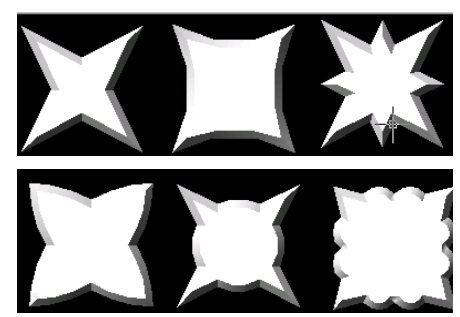

Figure 2. Some voxel elements

\subsection{Modeling Parameters}

A variety of voxels can be generated with the appropriate definitions of modeling parameters. A voxel is described with signature which is a set of modeling parameters and represented as $\mathrm{V}-\mathrm{Pt} / \mathrm{L} / \mathrm{T} / \delta / \mathrm{X} / \mathrm{Y}$. All of these modeling parameters are illustrated in Figure 3 and described as follows:

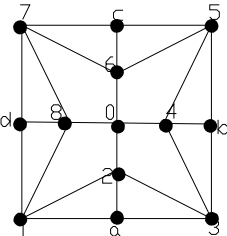

(a)

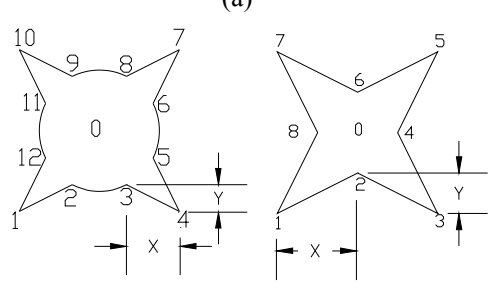

(b)
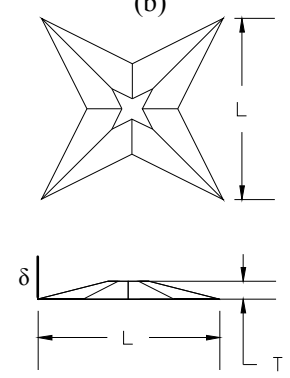

(c)

Figure 3. Modeling Parameters (a) Valid oints (b) Variants

(c) Dimensions of voxel and inclination angle of side surface

1. Number of valid points $(\mathrm{Pt})$ : It represents the maximum number of valid points from which voxels are defined. It may be nine, thirteen, or seventeen. The cross section of voxel is inscribed in a square with four corner and a center fixed valid points. The rest of the valid points are kept variables.
2. Size of voxel (L): It is equal to the size of square in which the voxel is inscribed.

3. Thickness of voxel (T): It is the thickness of voxel equal to depth of translational sweep. This parameter should be such that when thermoplastic (PET) material is blown with forming tool (blow mould); it would not be sheared off. So it needs to be carefully worked out.

4. Inclination of side surface $(\delta)$ : It is equal to taper angle of translational sweep to make the voxel sides inclined. This angle acts as draft which helps in easy removal of thermoplastic bottle out of mould decorated with patterns generated from voxels.

5. Variants (X and $\mathrm{Y}$ ): These are defined as distances between fixed point and next/previous variable point in horizontal and vertical direction respectively. Variants are used to achieve different shapes of cross sections resulting into different voxels.

\subsection{Modeling of Patterns}

The work presents area filling patterns as shown in Figure 4, which are generated by combining multiple copies of a voxel element in a rectangular array. The size of the pattern is defined by a number of rows and columns of the selected voxel, representing the size of rectangular array. The size of pattern is taken according to the area of middle section of the mould to be decorated.

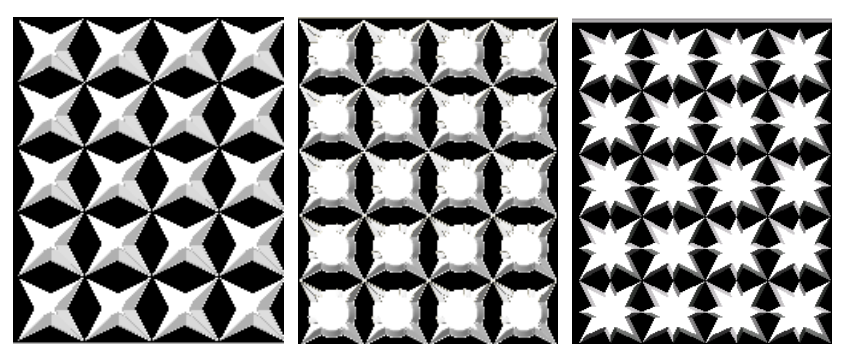

Figure 4. Area filling patterns

\section{TOROIDAL BENDING OF THE PATTERN}

The ornamental pattern is bent into toroidal (revolved) shape of bottle mould half cavity. This bending feature rotates each of the parallel planes around the intersection of the neutral plane and the end surfaces of the pattern by an angle specified. The neutral plane defines the theoretical plane of zero deformation (elongation or compression) along the sectional thickness of the bent pattern. The following steps are carried out for bending of pattern into the shape of bottle mould half cavity.

1. Step 1: The references shown in Figure 5 are generated which include a user coordinate system at the centeroid of the pattern, a sketching plane (SP) in X-Z plane passing through the centroid of the pattern to sketch the sectional bend profile, a reference plane (RP) perpendicular to the sketching plane, a neutral plane (NP) perpendicular to end planes passing through the centeroid of the pattern and two end planes (EP) parallel to the sketching plane at end surfaces of the pattern to define the radius of the toroid.

2. Step 2: The bend profile which defines the shape of the cross section of the toroid is created from a chain of entities (spline, arc, line, etc.). A 3-point arc is selected as bend profile shown in Figure 5. Two end points of the arc are fixed on extreme surfaces of the pattern and lie in the sketching plane. The third point lie in the $\mathrm{Z}$-axis and defines the radius and centre of bending curvature. 
The pattern is first bent along the bend profile about the bending axis passing through the center of curvature as shown in Figure 6. 3. Step 3: The two end planes are used to bend toward each other at an angle equal to 180 degrees to generate the shape of bottle mould half cavity. These planes pull the bent pattern toward each other and all geometry will be toroidally bent as shown in Figure 7.

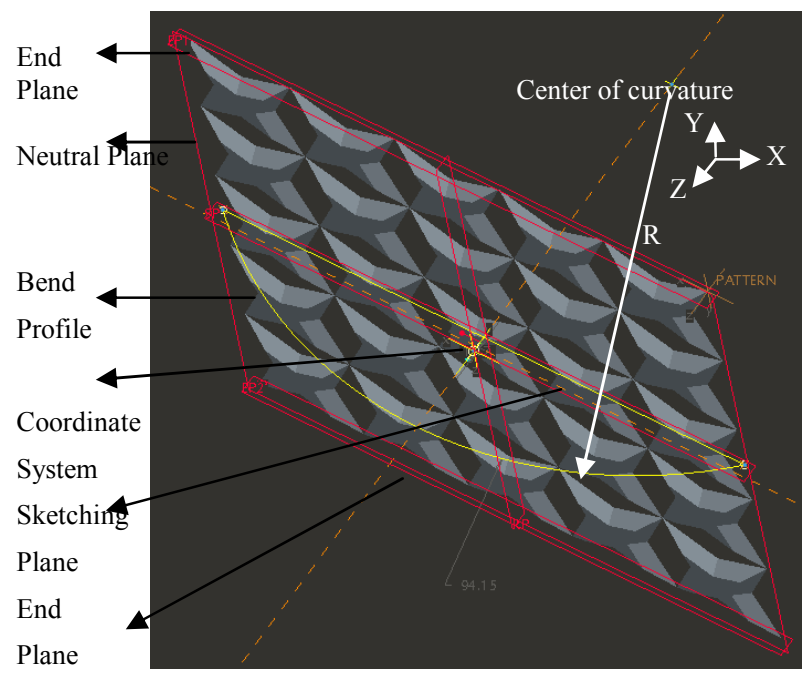

Figure 5. References

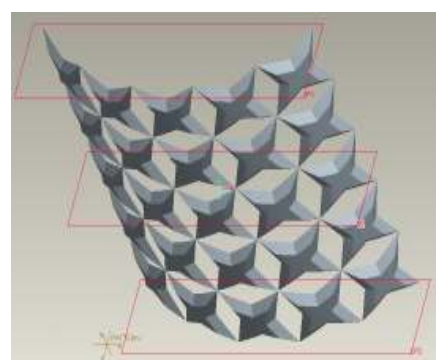

Figure 6. Bending along the bend profile

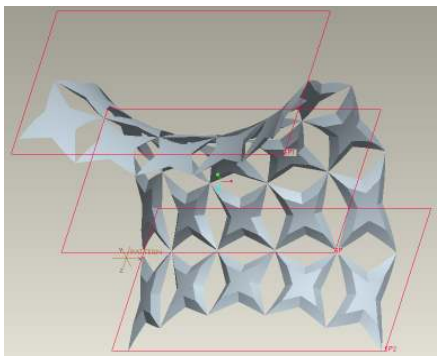

Figure 7. Toroidal bending at 180 degrees

\section{MODELING OF THE DECORATED BOTTLE MOULD}

The stock material is added to the toroidally bent pattern using the Variable Section Sweep feature to produce the decorated middle section of bottle mould half. The section trajectories are generated longitudinally across the variable cross sections of toroidally bent pattern to produce the mould cavity as shown in Figure 8 . The cross section of stock solid is created and swept along the selected trajectories as shown in Figure 9. The sketch of cross section does not change its shape as its being swept along the trajectories. The CAD model of the decorated middle section of bottle mould is rendered as shown in Figure 10 and used for post processing (generation of cutter location file).

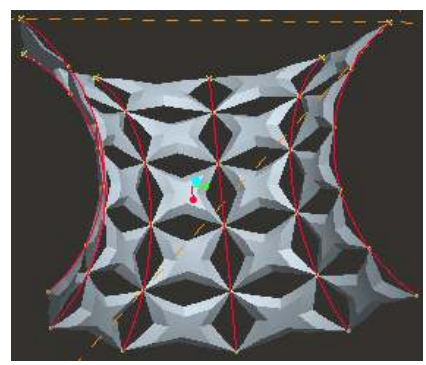

Figure 8. Generation of trajectories

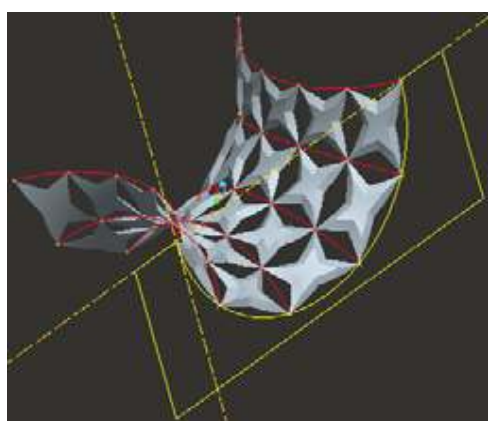

Figure 9. Creation of Cross Section of the Stock-Solid

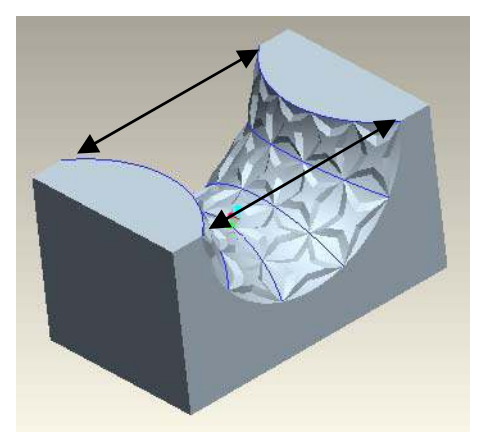

Figure 10. CAD model of middle section of the mottle mould half

The top and bottom sections of the mould half cavities take the shape of top portion of bottle dome and base of bottle each. These sections are created using the sketching approach which is shown 
in Figure 11. These sections are generated with two-dimensional entities to sketch a sweep base followed by rotational sweep about an axis to an angle of 180 degrees. The dimensions and profiles of the sweep bases to create top and bottom mould sections are shown in Figure 12 and taken according to the dimensions ' $R_{1}$ ' and ' $\mathrm{a}$ ' of the middle section of mould shown in Figure 10. The radius $R_{2}$ in the profile of top section is taken equal to the radius of threaded portion of bottle.

The three sections are aligned using and joined into the shape of decorated bottle mould half using the 'mate' constraint of CAD system as shown in Figures 13 and 14 respectively. The profile of middle section can be changed by altering the curvature of bend profile. Figure 14 shows two different profiles of middle section of bottle mould halves decorated with same pattern but generated with different bend radius of a 3-point arc used as bend profile.

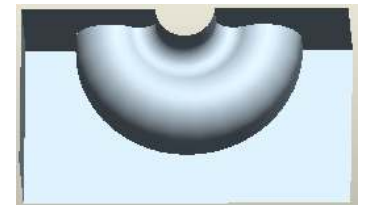

Top Section

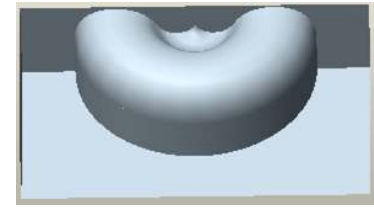

Bottom Section
Figure 11: Top and bottom sections of the mould half

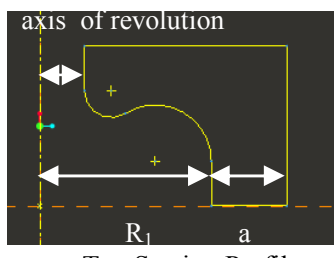

Top Section Profile

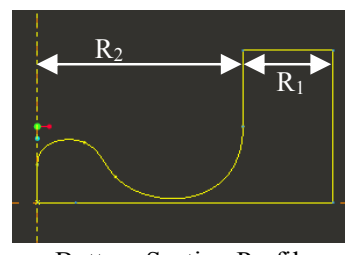

Bottom Section Profile
Figure 12: Sweep bases to create top and bottom sections of the mould half

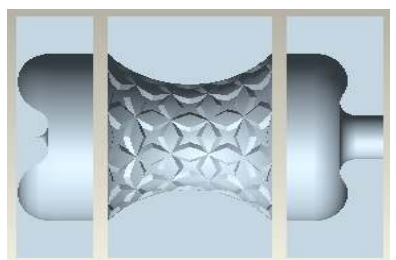

Figure 13. Alignment of three sections of the mould half
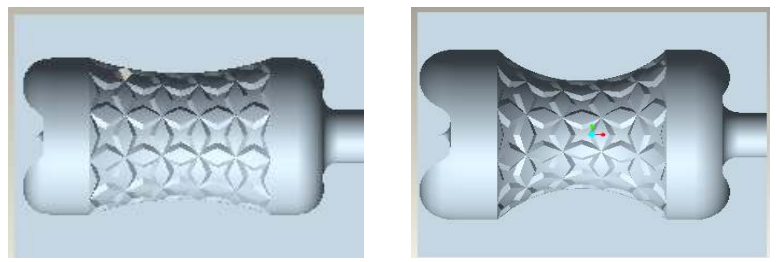

Figure 14. Different profiles of middle section decorated with same pattern and generated with different curvature of bend profile

\section{CONCLUDING REMARKS}

A novel CAD paradigm is presented to develop and decorate blow forming tools (mould) for producing PET bottles. This work is in the direction of integrated product development, envisages automating design and manufacturing of decorated moulds which, in turn, are utilized to blow PET bottles. The integrated design and manufacturing tools simplify the iterative development process and shorten the development time. The paradigm also provides the capability of designing custom-engineered bottle moulds using parametric design concept.

The geometric modeling techniques used in this work are simple, yet their results are very beautiful and inspiring. This CAD paradigm has been tested by creating STL format of a model and sending it to LMT machine for rapid tooling. Figure 15 shows a LMT model of bottle mould half. The CAD model of a bottle mould half is also used to generate the Cutter Location Data necessary to drive CNC machine tool to manufacture it from a rectangular block (shown in Figure 16 with green color) representing the raw stock. The simulated mould half sections are shown in Figure 16, which have been created using the CAM system directly from 3D CAD model.

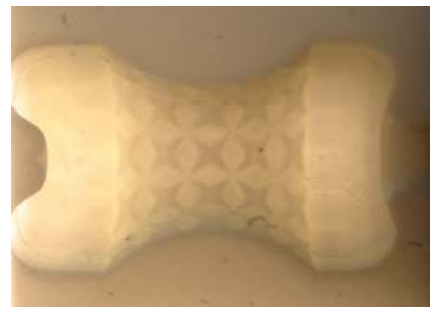

Fig. 15. LMT model of the bottle mould half
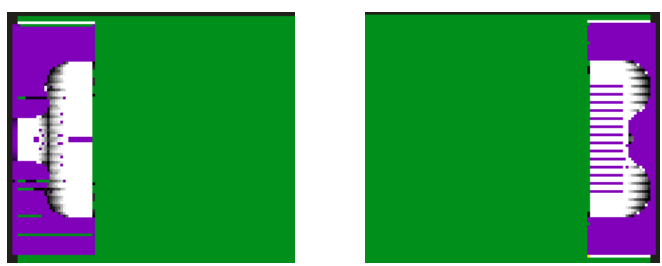

(a)
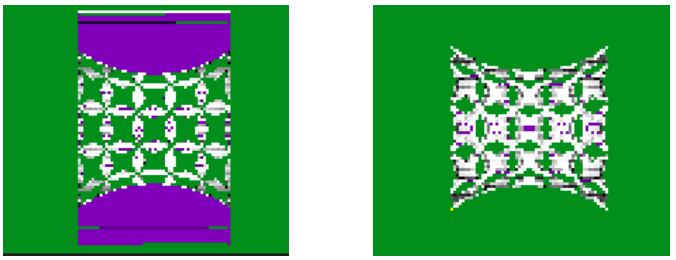

(b)

Figure 16. Simulated mould half sections (a) Top and bottom (b) Middle 


\section{REFERENCES}

[1] Chua C. K. and Lye S. L., 1998, Parametric modeling of drinking bottles", Integrated Manufacturing Systems, MCB University Press, 9, 2, 99-108.

[2] Johnston R. R., Czesak T. M., Scott A. J. and Beaufils I. F., Dec. 9, 2000, Prototype mold for blow molding hollow plastic containers and method of making same", U.S. Patent 6116888 .

[3] William J. C. and Patrick J. F. E., 2007, Injection stretch blow molded container, U.S. Patent 20070048473.

[4] Yourist S. E., Jan 6, 2004, Method of designing and manufacturing a plastic blow-molded container, U. S. Patent 6673281.

[5] Tam K. W. and Chan K. W., 2005, Case Study: A Novel Surface Scanning System, Journal of Robotic Systems, Willey Inter Science, 22, 7, 359-366.

[6] Gulati V. and Tandon P., 2007, A parametric voxel oriented CAD paradigm to produce forming components for stretch formed jewelry", Computer-Aided Design \& Applications, $4,4,137-145$. 